\title{
Reducing Customers' Total Cost of Ownership Within a Software Supply Network
}

\author{
Slinger Jansen \\ Information Sciences Institute \\ Utrecht University \\ Utrecht, the Netherlands \\ slinger.jansen@cs.uu.nl
}

\author{
Wilfried Rijsemus \\ Marketing and Product Management \\ Cordys Europe \\ Putten, the Netherlands \\ wrijsemus@cordys.com
}

\begin{abstract}
This paper describes how the company Cordys avoids the ERP problems from the last 15 years by improving the software release, delivery, deployment, and maintenance processes. These ERP problems, such as costly ERP migrations and highly complex maintenance procedures, are circumvented by the introduction of a staging area. This staging area is a software control system that enables a customer to manage software, $3 r d$ party/home developed products, and knowledge such as manuals, licenses and updates, explicitly.
\end{abstract}

\section{Introduction}

Organisations have suffered many maintenance problems with their Enterprise Resource Planning (ERP) applications. These business critical applications are hard if not costly to update, impossible to test in all possible configurations, cost intensive to support, and present a total cost of ownership that is disproportionate to the purchase price. This paper discusses how the software vendor Cordys aims to avoid the problems associated with the ERP lessons of the last fifteen years. Cordys' solutions and novel view on software maintenance are presented and provide the reader an insight into ERP 2.0.

Throughout the 1990s and early 2000s ERP systems, which (attempt to) integrate all data and processes of an organization into a single unified system, quickly flooded the markets. As these systems became more important to organizations the demand for flexibility, customisations, support, and quick releases with new features increased. These systems proved unstable, inflexible, and often colossal to its end-users and managers. The largest amount of problems for ERP products however, was and still is found in maintenance. Business managers often demand new features on short notice, wanting to migrate often and quickly to stay agile in a changing business environment. Simultaneously these growing ERP systems become harder to maintain and update for system managers, due to the large amounts of customisations, data, and third-party applications that make up the system. For the software vendors too, life has become too complex to manage. Their multitudes of software releases, 3rd party components, and dependencies amongst components cause the amount of tests that need to be run to explode in number. Also, even if the vendor is aware of a customer's configuration, the vendor is often unable to simulate the customer's environment due to the large amount of customisations created on the customer side.

The ERP market clearly shows that a paradigm-shift is taking place for ERP and software products in general. Whereas in the past software was delivered to customers directly from the vendor, including third party components, it is now not uncommon to have a large number of parties involved that perform customisation, development, and maintenance on a software product. This leads to a shift from software supply chains, to software supply networks. In these networks a software vendor can, for example, deliver its ERP product, together with a number of third-party extensions to a customer. The customer now deploys the product, purchases another third-party customisation, and performs some customisation itself. A software supply network thus is defined as a network that aims for participants (vendors and customers) to jointly create competitive advantage from diverse sources for themselves and for others.

Cordys (http://www.cordys.com) is a software vendor that sells products that apply a virtual communication layer on top of installed systems and processes to international customers from medium to very large size. Customers include banks, insurance companies, logistics companies, and many others. Currently Cordys employs over 550 people, of which approximately 325 are based in India. The Cordys Enterprise Service Bus (ESB) provides a platform for ERP applications to communicate using UDDI (Universal Description, Discovery and Integration protocol for web services) and SOAP for interaction. New systems can be added and processes can be developed and added without having 
to modify or remove any existing systems. The Cordys ESB can also be used in conjunction with other products to connect (legacy) ERP applications and publish these on-line. Cordys is a relatively young player in the industry, having been established in 2001, however, Cordys has brought along 20 years of experience from earlier products, such as the successful Baan ERP suite.

\section{There is no "Single Customer"}

To solve the issues presented in the introduction Cordys has introduced the Staging Area (SA), an application that is deployed on a customer system before the actual ESB is deployed. The SA is used to manage software applications and knowledge about these software applications, such as manuals, updates, and licenses. The SA supports multiple deployments, to allow for development, test, acceptance, and production environments enabling customers to design their internal update and development processes using the Information Technonology Infrastructure Library (http://www.itil.co.uk) standards. Customers need customised release procedures, because they themselves too develop extensions and customizations on the Cordys Platform that must be merged with Cordys' releases. The staging area thus contains knowledge about the customer's configuration and can download manuals, licenses, and updates specifically for one customer's configuration. The staging area solves three problems for Cordys.

The first problem is the ubiquitous conflict within customer organizations. On the one hand, there is a system manager who wants business critical systems to be as stable as possible without any downtime or maintenance time. On the other there is the business manager who wants to remain as flexible as possible with new features and bug fixes. Many software vendors in the past experienced that customers would lag behind several months or even years, simply because they could not afford to update due to high upgrade and migration costs. Cordys and its SA enable a model where customers can decide themselves when to update the production server, based on real information from the test, development, and acceptance servers. In order to convince customers to update, Cordys does guarantee its customers a specified quality level to build up a trust relationship.

The second problem solved by the SA is that finding and implementing a bug fix (that might have even been released a year ago) may take months. The SA is now used for sending updates to the customer, but only those that are relevant for the customer, such that the system manager does not need to search through numerous forums to find new fixes on a weekly basis. Also, when a problem does occur, Cordys can download the current state of the system (if the customer allows Cordys to) for further evaluation. Cordys has assembled a number of rules to establish whether a configuration is valid or not. If the configuration is indeed valid, it will be simulated on a virtual environment at the support department to find the cause of a bug. This process takes a maximum of ten minutes, allowing support engineers to reproduce and find errors quickly.

A third problem that the SA has solved for Cordys is the problem that software and surrounding knowledge are always out of sync. Manuals might be outdated, fixes are only valid for a limited range of revisions, and customisations will only work on so many different configurations. For Cordys this problem becomes apparent due to the fact that they operate in a software supply network, where many different parties can build ESB applications, customisations, and extensions. Cordys' solution to this is to have a very strict versioning management system, where knowledge, customisations, and extensions are all versioned along with major components. This forces developers to keep all development activities under strict configuration management. The pay-off comes quickly though, due to the fact that quality guarantees can be given based upon this information.

\section{Discussion}

A large number of third-party developers and customers doing their own development on the Cordys platform require descriptive development strategies from Cordys, making these strategies part of the product itself. Still, Cordys does not see this as a similar situation as large numbers of groups working for an ERP product. Whereas in the past ERP vendors were pompously "orchestrating the market" Cordys feels that it is merely "beating the drum".

Besides the fact that Cordys has proven to be a successful player on the ERP market, the question remains whether Cordys' solutions actually provide features to grow to ERP 2.0, or whether Cordys merely hums an old tune. It is our firm belief that for ERP to grow into a new generation, the next step relies on integration and quick incremental evolution of functionality [1]. The SA as presented by Cordys, suffices because it enables multiple deployments, is used to gain information about the customer's configuration, and it can quickly evolve installed applications in accordance with the customer's and Cordys' required speed, reducing migration, extension, and upgrading costs.

\section{References}

[1] S. Jansen and S. Brinkkemper. Definition and validation of the key process areas of release, delivery and deployment of product software vendors: turning the ugly duckling into a swan. In proceedings of the International Conference on Software Maintenance (ICSM2006, Research track), September 2006. 